Article

\title{
Solvability of nonlinear equations in case of branching solutions
}

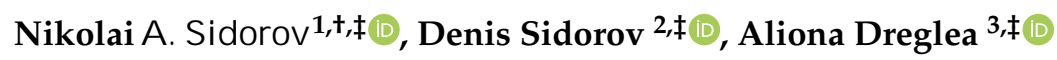 \\ Institute of Mathematics and Information Technologies, Irkutsk State University; sidorovisu@gmail.com \\ Energy Systems Institute, Russian Academy of Sciences; dsidorov@isem.irk.ru \\ Baikal School of BRICS, Irkutsk National Research Technical University; adreglea@gmail.com \\ * Correspondence: sidorovisu@gmail.com (N.S.) \\ $\ddagger \quad$ These authors contributed equally to this work.
}

\begin{abstract}
The necessary and sufficient conditions of existence of the nonlinear operator equations' branches of solutions in the neighbourhood of branching points are derived. The approach is based on reduction of the nonlinear operator equations to finite-dimensional problems. Methods of nonlinear functional analysis, integral equations, spectral theory based on index of Kronecker-Poincaré, Morse-Conley index, power geometry and other methods are employed. Proposed methodology enables justification of the theorems on existence of bifurcation points and bifurcation sets in the nonstandard models. Formulated theorems are constructive. For a certain smoothness of the nonlinear operator, the asymptotic behaviour of the solutions is analysed in the neighbourhood of the branch points and uniformly converging iterative schemes with a choice of the uniformization parameter enables the comprehensive analysis of the problems details. General theorems are illustrated on the nonlinear integral equations.
\end{abstract}

Keywords: branch points; bifurcation points; Fredholm operator; uniformization; asymptotics; iterations; regularization

\section{Introduction}

The progress in the methods of analysis development and the new nonlinear problems of applied mathematics enable the nonlinear functional analysis novel concepts formulation. In the seminal paper of L.A. Lusternik [1] the main directions of this field paved the anevue for the branching theory of nolinear equations development. The classic works of A.M. Lyapunov, A.I. Nekrasov, J.H. Poincaré, M.A. Krasnoselsky [2], J. Toland and others contributed to this field. In their studies, reductions of the given nonlinear models to the finite-dimensional systems with parameters were used. The finite-dimensional equivalent system is now known as Lyapunov-Schmidt branching system and the corresponding method is known as Lyapunov-Schmidt (LS) method. In the review [3] and monograph of M.M. Vainberg and V.A. Trenogin [4] the basement of the analytical theory of branching solutions in Banach spaces with applications is given. These works contributed to the modern functional analysis development with many applications [5-22].

Studies of the integral equations in mechanics played the principal role not only in LS method development, but also iniciated the way for functional analysis new chapters construction. Studies of integral equations' bifurcation points are necessary in various mathematical models in the various fields. For example, for some parameter values there the loss of stability may occur and another series of solutions branches off from that bifurcation point.

Such problems include both classical problems of the critical load of the rod and the formation, the emergence of new forms of equilibrium of rotating fluids, a branch at a critical wind speed from 
the horizontal surface of the waves, and a number of novel challenging bifurcation problems in biochemistry, plasma physics, electrical engineering and many other applied fields. Over the past decade, the branching theory of solutions of nonlinear equations with parameters and its applications have received enormous development and practical applications.

The monographs [17,23] and papers [24-32] review of the recent results and number of applications in this fields are given. The special attention has been paid to the theory development in term of the Sobolev-Schwartz theory of distributions [33-35]. Applications of group methods [36] in bifurcation theory are given in $[8,13,16,25,26,37]$.

Despite the abundance of literature in the last 20 to 30 years and interesting results focused on the theory of branching solutions, the formulation and proof of the general existence theorems in nonlinear non-standard models with parameters is still an open problem. The problem of approximate methods development in the neighborhood of critical points ia still open. The clarity of the methods and results presentation using the elementary methods is also important. The objective of this article to fill the gap between abstract theory development and concrete problems solution.

It is to be noted that only some part of the total set of results in this field we discuss due to the limited size of the article. Applications and many other outstanding results including cosymmetry by Yudovich, projective-iterative techniques, center manifold reduction, global existence theorems, have remained beyond its scope.

The remainder of this paper is structured as follows. Sec. 2 demonstrates the construction of the main part of the branching Lypunov-Schmidt equation and its analysis. The existence theorems of bifurcation points and bifurcation manifolds of real solutions are proved. These theorems generalizes the numbder of well-known theorems on bifurcation points. Examples of solving integral equations with bifurcation points and points of enhanced bifurcation are given.

Methods for parameterizing the branches of solutions of nonlinear equations in a neighborhood of branch points are described in Sec. 3 and Sec. 4. Iterative methods for constructing branches with the choice of a uniformization parameter are provided that ensure uniform convergence of iterative schemes in the neighborhood of the critical parameter values. Concluding remarks, regularization and generalizations for interwined equations are provided in Sec. 5.

\section{Existence Theorem of Bifurcation Points and Manifolds of Nonlinear Equations}

Let $X, Y$ are real Banach spaces, $\Lambda$ is real normed space. We consider the equation

$$
B x=R(x, \lambda),
$$

where $B: D \subset X \rightarrow Y$ is closed Fredholm operator with dense domain $D, \lambda \in \Lambda$. Nonlinear operator $R(x, \lambda)$ with values in $Y$ is defined, continuous, and continuosly differentiable in Fréchet sense wrt $x$ in the neighborhood

$$
\Omega=\{x \in X, \lambda \in \Lambda \mid\|x\|<r,\|\lambda\|<\rho\} .
$$

We assume that equation (1) has trivial solution $x=0$ for all $\lambda$ and

$$
R(0, \lambda)=0, R_{x}(0,0)=0
$$

Definition 1. Point $\lambda=0$ is called bifurcation point of equation (1) if in any neighborhood of point $x=$ $0, \lambda=0$ exists pair $(x, \lambda)$ for $x \neq 0$ which satisfies equation (1).

Corollary 1. If equation $B x=R(x, 0)$ has nonisolated trivial solution $x=0$ then $\lambda=0$ will be bifurcation point for equation (1). 
Definition 2. Point $\lambda=0$ is called as the strong bifurcation point of equation (1) if in the arbitrary neighborhood of pair $x=0, \lambda=0$ exists pair $(x, \lambda)$ such as $x \neq 0, \lambda \neq 0$ which satisfies equation (1).

Corollary 2. If $\lambda=0$ is strong bifurcation point, then $\lambda=0$ is bufurcation point.

Theorem 1. If order for the point $\lambda=0$ to be a bifurcation point, it is necessary that homogenius linear equation $B x=0$ has nontrivial solution.

Proof. If equation $B x=0$ has only trivial solution, then Fredholm operator $B$ has bounded inverse $B^{-1}$ and equation (1) can be reduced to equation

$$
x=B^{-1} R(x, \lambda)
$$

which meets the condition of the contraction mapping principal in the small neighborhood of pair $x=0, \lambda=0$. Therefore, equation enjoys unique solution in that neighborhood. Because of improsed conditions $R(0, \lambda)=0$ equation (2) has only trivial solution in the small neighborhood of the point $x=0, \lambda=0$. Theorem is proved.

Let us now focus on the sufficient conditions of bifurcation points existence. We introduce the basis $\left\{\phi_{i}\right\}_{1}^{n}$ in subspace $N(B)$, basis $\left\{\psi_{i}\right\}_{1}^{n}$ in $N\left(B^{*}\right)$ and system $\left\{\gamma_{i}\right\}_{1}^{n} \in X^{*},\left\{z_{i}\right\}_{1}^{n} \in Y$ which are biorthogonal to these basises, i.e.

$$
\begin{aligned}
& \left\langle\phi_{i}, \gamma_{k}\right\rangle= \begin{cases}1, & i=k \\
0, & i \neq k\end{cases} \\
& \left\langle z_{i}, \psi_{k}\right\rangle= \begin{cases}1, & i=k \\
0, & i \neq k .\end{cases}
\end{aligned}
$$

Then from the Schmidt - Trenogin Lemma (see Lemma 2.1.1. [? ]) it follows that operator $\hat{B}=$ $B+\sum_{i=1}^{n}\left\langle\cdot, \gamma_{i}\right\rangle z_{i}$ is contunuosly invertible. Let $\Gamma=\hat{B}^{-1}$. Then $\Gamma z_{i}=\phi_{i}, i=1,2, \ldots, n$. Let us introduce the projectors

$$
\begin{aligned}
& P=\sum_{i=1}^{n}\left\langle\cdot, \gamma_{i}\right\rangle \phi_{i}, \\
& Q=\sum_{i=1}^{n}\left\langle\cdot, \psi_{i}\right\rangle z_{i}
\end{aligned}
$$

and direct decompositions $X=X_{n} \dot{+} X_{\infty-n}, Y=Y_{n} \dot{+} Y_{\infty-n}$, where $X_{n}=P X=N(B), X_{n-\infty}=$ $(I-P) X, Y_{n}=Q Y=\operatorname{span}\left\{z_{1}, \cdots, z_{n}\right\}, Y_{\infty-n}=(I-Q) Y=R(B)$. Obviusly, $X_{\infty-n}=\{x \in X:$ $\left.\left\langle x, \gamma_{i}\right\rangle=0, i=1, \ldots, n\right\}, Y_{\infty-n}=\left\{y \in Y:\left\langle y, \psi_{i}\right\rangle=0, i=1, \ldots, n\right\}$. Let us rewrite eq. (1) as following system

$$
\begin{aligned}
& \hat{B} x=R(x, \lambda)+\sum_{s=1}^{n} \xi_{s} z_{s} \\
& \xi_{s}=\left\langle x, \gamma_{s}\right\rangle, s=1, \ldots, n .
\end{aligned}
$$

Equation (3) by multiplication with operator $\Gamma$ can be reduced to

$$
x=\Gamma R(x, \lambda)+\sum_{s=1}^{n} \xi_{s} \phi_{s} .
$$

Using change

$$
x=\sum_{s=1}^{n} \xi_{s} \phi_{s}+u(\xi, \lambda)
$$


eq. (5) can be reduced to the following equation

$$
u(\xi, \lambda)=\Gamma R(\xi \phi+u(\xi, \lambda), \lambda) .
$$

For sake of clarity let us assume $\sum_{s=1}^{n} \xi_{s} \phi_{s}=\xi \phi$. For arbitrary $\xi$, $\lambda$ from small neighborhood of origin due to contraction mapping principal the sequence $u_{m}=\Gamma R\left(\xi \phi+u_{m-1}, \lambda\right), u_{0}=0$ converges to unique solution $u(\xi, \lambda)$ of equation (7). In that case $u(0, \lambda)=0$. Because of

$$
\frac{\partial u}{\partial \xi_{i}}=\Gamma R_{x}(\xi \phi+u, \lambda)\left(\phi_{i}+\frac{\partial u}{\partial \xi_{i}}\right)
$$

then, taking into account function $u$ continuity wrt $\xi, \lambda$ and equality $R_{x}(0,0)=0$ in the small neighborhood of origin, we get the following equality

$$
\frac{\partial u}{\partial \xi_{i}}=\left[I-\Gamma R_{x}(\xi \phi+u, \lambda)\right]^{-1} \Gamma R_{x}(\xi \phi+u, \lambda) \phi_{i}, i=1, \ldots, n .
$$

This formula can be presented as follows

$$
\frac{\partial u}{\partial \xi_{i}}=\sum_{n=1}^{\infty}\left(\Gamma R_{x}(\xi \phi+u, \lambda)\right)^{n} \phi_{i}
$$

as $\left\|R_{x}(\xi \phi+u, \lambda)\right\| \leq q<1$. Therefore, using the Taylor formula, the desired function $u(\xi, \lambda)$ in the problem of bifurcation point search in the neighborhood of point $\xi=0$ can be represented as following series

$$
u(\xi, \lambda)=\sum_{k=1}^{\infty}\left(\Gamma R_{x}(0, \lambda)\right)^{k} \sum_{s=1}^{n} \xi_{s} \phi_{s}+r(\xi, \lambda),
$$

where $\|r(\xi, \lambda)\|=o(\|\xi\|)$. Taking into account (4), (6), (7), the following finite-dimentional branching system of Lyapunov-Schmidt (LS) can be derived

$$
L_{k}(\xi, \lambda):=\left\langle\sum_{i=1}^{\infty}\left(\Gamma R_{x}(0, \lambda)\right)^{i} \sum_{s=1}^{n} \xi_{s} \phi_{s}+r(\xi, \lambda), \gamma_{k}\right\rangle=0, k=1, \ldots, n .
$$

Taking into account equality $\Gamma^{*} \gamma_{k}=\psi_{k}$, branching system (8) can be presented as follows

$$
\sum_{s=1}^{n}\left\langle R_{x}(0, \lambda)\left(I-\Gamma R_{x}(0, \lambda)\right)^{-1} \phi_{s}, \psi_{k}\right\rangle \xi_{k}+\rho_{k}(\xi, \lambda)=0, k=1,2, \ldots, n
$$

or, briefly, in the matrix form

$$
L(\xi, \lambda):=M(\lambda) \xi+b(\xi, \lambda)=0 .
$$

Here $M(\lambda)=\left[\left\langle R_{x}(0, \lambda)\left(I-\Gamma R_{x}(0, \lambda)\right)^{-1} \phi_{s}, \psi_{k}\right\rangle\right]_{s, k=1}^{n}, b(\xi, \lambda)=\left(\rho_{1}(\xi, \lambda), \cdots, \rho_{n}(\xi, \lambda)\right)^{T},\|b\|=$ $o(\| \xi||)$. Let us employ matrix $M(\lambda)$ to get the sufficient conditions for point $\lambda=0$ to be such a bifurcation. We introduce the set $\{\lambda \in \Lambda$ : $\operatorname{det} M(\lambda)=0\}$ which contain the possible bifurcation point $\lambda=0$.

Let us introduce the condition

Condition 1. Let in the neighborhood of point $\lambda=0$ there exists set $S$ which is Jordan continuum, and $S=S_{+} \cup S_{-}$and $0 \in \partial S_{+} \cap \partial S_{-}$. There let exists continuous mapping $\lambda(t)$ as $t \in[-1,1]$ with values in $S$ such as $\lambda:[-1,0) \rightarrow S_{-}, \lambda:(0,1] \rightarrow S_{+}, \lambda(0)=0$. Moreover, let $\operatorname{det} M(\lambda)=\alpha(t)$, where $\alpha(t):[-1,1] \rightarrow \mathbb{R}^{1}$ is continuous function which is zero only for $t=0$. 
Theorem 2. (Sufficient bifurcation condition) Let condition 1 be fulfilled, where $\alpha(t)$ is monotone increasing function. Then $\lambda=0$ is bifurcation point of eq. (1). Moreover, if trivial solution of equation $B x=R(x, 0)$ is isolated, then $\lambda=0$ will be such stronger bifurcation.

Proof. Let $\lambda=\lambda(2(\theta-1) \delta)$ in branching system (21) for arbitrary small $\delta>0$ and $\theta \in[0,1]$ and consider the continuous vector field

$$
H(\xi, \theta):=\mathcal{L}(\xi, \lambda((2 \theta-1) \delta)): \mathbb{R}^{n} \times \mathbb{R}^{1} \rightarrow \mathbb{R}^{n}
$$

defined for $\xi, \theta \in M$, where $M=\{\xi, \theta \mid\|\xi\|=r, 0 \leq \theta \leq 1\}, r>0$ is as small as possible.

Case 1. Let us assume $H\left(\xi^{*}, \theta^{*}\right)=0$ where $\left(\xi^{*}, \theta^{*}\right) \in M$. Then by Definition $1 \lambda=0$ will be bifurcation point of eq. (1).

Case 2. Let us assume $H(\xi, \theta) \neq 0$ for $\forall(\xi, \theta) \in M$ and consequently null of the space $\Lambda$ is not bifurcation point. Then, due to continuity, fields $H(\xi, 0), H(\xi, 1)$ are homotopic on the sphere $\|\xi\|=r$. Then its rotations are equal:

$$
J(H(\xi, 0),\|\xi\|=r)=J(H(\xi, 1),\|\xi\|=r)
$$

Due to condition 1 and assumption $H(\xi, \theta) \neq 0$ for $\forall(\xi, \theta) \in M$ fields $H(\xi, 0), H(\xi, 1)$ are homotopic to their linear parts $M(\lambda(-\delta)) \xi, M(\lambda(+\delta)) \xi$. Then

$$
\begin{aligned}
& J(H(\xi, 0),\|\xi\|=r)=J(M(\lambda(-\delta) \xi),\|\xi\|=r), \\
& J(H(\xi, 1),\|\xi\|=r)=J(M(\lambda(+\delta) \xi),\|\xi\|=r) .
\end{aligned}
$$

Because det $M(\lambda)=\alpha(t)$ then using index Konecker the following equalities are fulfilled

$$
\begin{aligned}
& J(M(\lambda(-\delta) \xi),\|\xi\|=r)=\operatorname{sign} \alpha(-\delta), \\
& J(M(\lambda(+\delta) \xi),\|\xi\|=r)=\operatorname{sign} \alpha(+\delta) .
\end{aligned}
$$

Due to Condition $1, \alpha(-\delta)<0, \alpha(+\delta)>0$ then equality (10) is not satisfied and therefore there exists point $\left(\xi^{*}, \theta^{*}\right) \in M$ for which $H\left(\xi^{*}, \theta^{*}\right)=0$ and point $\lambda=0$ is bifurcation point for eq. (1). Theorem 1 is proved.

Corollary 3. Let condition I is fulfilled for $\forall \lambda \in \Omega_{0}$. Then $\Omega_{0}$ will be bifurcation set of eq. (1). If in that case $\Omega_{0}$ is connected set and each points belongs to the neighborhood of homeomorphic some set of space $\mathbb{R}^{n}$ then $\Omega_{0}$ will be n-dimentional manyfolds of bifurcation of eq. (1).

From Theorem 1 it follows the known streithern of the known Theorem of M.A. Krasnoselsky on bifurcation point of odd mutiplicity.

Definition 3. System of branching equations of Lyapunov-Schmidt we call potential if $L(\xi, \lambda)=\operatorname{grad}_{\xi} v(\xi, \lambda)$.

Obviosly, (8) is potential system if matrix $\left[\frac{\partial L_{k}(\xi, \lambda)}{\partial \xi_{i}}\right]_{i, k=1}^{n}$ is symmetric for $\forall(\xi, \lambda)$ from neighborhood of null. Let us outline that

$$
\left[\frac{\partial L_{k}}{\partial \xi_{i}}\right]_{i, k=1}^{n}=\left\langle R_{x}(\xi \phi+u(\xi, \lambda), \lambda)\left[I-\Gamma R_{x}(\xi \phi+u(\xi, \lambda), \lambda)\right]^{-1} \phi_{i}, \psi_{k}\right\rangle_{i, k=1}^{n} .
$$

Then we have the following lemma on potentiality of eq. (9) 
Lemma 1. The branching equation (9) is potential if and only if all the matices

$$
\left[\left\langle R_{x}\left(\Gamma R_{x}\right)^{m} \phi_{i}, \psi_{k}\right\rangle\right]_{i, k=1}^{n}
$$

$m=0,1, \ldots$ are symmetric in the neighborhood of $(0,0)$.

Corollary 4. Let $X=Y=H, H$ is Hilbert space. Let operator $B$ is symmetric in $D$, and operator $R_{x}(x, \lambda)$ is symmetric for $\forall(x, \lambda)$ from zero neighborhood. Then LS eq. (9) is potential.

Proof. In conditions of the symmetric operators $B$ and $R_{x}$ the equalities $\phi_{i}=\psi_{i}, i=1, \ldots, n$ are valid and

$$
\left\langle R_{x} \phi_{i}, \phi_{k}\right\rangle=\left\langle\phi_{i}, R_{x} \phi_{k}\right\rangle
$$

Since $\Gamma=\Gamma^{*}$, then

$$
\left\langle R_{x}\left(\Gamma R_{x}\right)^{m} R_{x} \phi_{i}, \phi_{k}\right\rangle=\left\langle\phi_{i}, R_{x}\left(\Gamma R_{x}\right)^{m} \phi_{k}\right\rangle
$$

for $m=1,2, \ldots$ Therefore, for arbitrary $\xi, \lambda$ from zero neighnborhood there following equalities are valid

$$
\frac{\partial L_{k}}{\partial \xi_{i}}=\frac{\partial L_{i}}{\partial \xi_{k}}, i, k=1, \ldots, n
$$

and LS is potential in sense of definition 3.

Let us find the corresponding potential $U(\xi, \lambda)$ of LS system. We introduce the notation

$$
a_{s k}(\lambda)=\left\langle R_{x}(0, \lambda)\left(I-\Gamma R_{x}(0, \lambda)\right)^{-1} \phi_{s}, \phi_{k}\right\rangle, s, k=1, \ldots, n
$$

Then branching system in the potential case is as follows

$$
L_{k}(\xi, \lambda):=\sum_{s=1}^{n} a_{s k}(\lambda) \xi_{s}+b_{k}(\xi, \lambda)=0, k=1, \ldots, n
$$

where $a_{s k}=a_{k s}, \frac{\partial b_{k}}{\partial \xi_{s}}=\frac{\partial b_{s}}{\partial \xi_{k}}, k, s=1, \ldots, n$. Therefore the desired potential will be function $U(\xi, \lambda)=$ $\frac{1}{2} \sum_{s, k=1}^{n} a_{s k}(\lambda) \xi_{s} \xi_{k}+W(\xi, \lambda)$ where $|w(\xi, \lambda)|=\mathcal{O}\left(\|\xi\|^{2}\right)$

Let us introduce

Condition II. Let LS eq. (9) is potential and let in the neighborhood of the $\|\lambda\|<\varepsilon$ there exists space $S$ containing the point $\lambda=0$ and it is Jordan continuum, $S=S_{+} \cup S_{-}, 0 \in \partial S_{+} \cap \partial S_{-}$. Let $\operatorname{det}\left[a_{j k}(\lambda)\right]_{\lambda \in S_{+} \cap S_{-}} \neq 0$ and matrix $\left[a_{j k}(\lambda)\right]$ for $\lambda \in S_{+}$has exactly $v_{1}$ negative eighenvalues, and for $\lambda \in S_{-}$has exactly $v_{2}$ negative eighenvalues.

Lemma 2. Let LS eq. (9) is potential, conditions II are fuilfilled, let $v_{1} \neq v_{2}$. Then for $\forall \varepsilon>0$ there exists $\lambda^{*}$ in the sphere $\|\lambda\|<\varepsilon$ such as the potential $v(\xi, \lambda)$ in the sphere $\|\xi\|<\varepsilon$ has stationary point $\xi \neq 0$.

Proof. (by contradiction) Let grad $v(\xi, \lambda) \neq 0$ as $0<\|\xi\|<\varepsilon,\|\lambda\|<\varepsilon$. Then based on homotopical invariance of the generalised index of Morse-Conley [? ] it is necessary $v_{1}=v_{2}$ and we observe the contradiction with conditions of Lemma 2.

Remark 1. In some special cases for $\lambda \in \mathbb{R}^{1}$ we have provided the analytical proofs of this lemma using the Rolle theorem, Morse lemma and local coordinates. Using Lemma 2 there following theorem on bifurcation points existence is valid. 
Theorem 3. Let LS eq.(9) is potential and let condition II be fulfilled for $v_{1} \neq v_{2}$. Then $\lambda=0$ is bifurcation point of equation (1). If in such a conditions $x=0$ is isolated solution of equation $B x=R(x, 0)$, then $\lambda=0$ will be the strong bifurcation point of eq. (1).

Proof follows from Lemma 1, Definitions 1, 2 and from Corollary 1.

Corollary 5. Let is condition II, $\Lambda=\mathbb{R}^{1}$, matrix $\left[\alpha_{i k}(\lambda)\right]$ as $\lambda \in(0, \varepsilon)$ is positive defined and symmetric matrix, and for $\lambda \in(-\varepsilon, 0)$ is negative defined and symmetric matrix. Then $\lambda=0$ is bifurcation point of equation (1).

Example 1. Let is consider the equation

$$
x(t)=\int_{a}^{b} K(t, s, x(s), \lambda) d s
$$

where $K(t, s, x, \lambda)=a(t) a(s) x(s)+\sum_{i=1}^{\infty} \lambda^{i} K_{i}(t, s) x(s)+o(\|x\|)$ as $|\lambda|<\rho,|x|<r$ and all functions are continuous. Let $\int_{a}^{b} a^{2}(t) d t=1$. Assume $X=Y=\mathcal{C}_{[a, b]}, \Lambda=\mathbb{R}^{1}$ and consider this equation as an abstract equation (1) such as $B x=x(t)-\int_{a}^{b} a(t) a(s) x(s) d s, R_{x}(0, \lambda) x=\int_{a}^{b} \sum_{i=1}^{\infty} \lambda^{i} K_{i}(t, s) x(s) d s$. Let

$$
\int_{a}^{b} \int_{a}^{b} K_{i}(t, s) a(t) a(s) d s d t=\left\{\begin{array}{l}
0, \text { if } i=1,2, \ldots, 2 m \\
c \neq 0, \text { if } i=2 m+1 .
\end{array}\right.
$$

Then correspinding LS is as follows $\left(c \lambda^{2 m+1}+o\left(\lambda^{2 m+2}\right)\right) \xi+o(|\xi|)=0$. Therefore, here branching system (9) contains the single equation where function $M(\lambda)=c \lambda^{2 m+1}+o\left(\lambda^{2 m+1}\right)$ changes sign after zero crossing. Therefore, Theorems 2 and 3 conditions are fulfilled and $\lambda=0$ is bifurcation point.

Under additional conditions on nonlinear functions in the integral eqaution the bifurcation point will be the strong bifurcation point and its nontrivial real solutions can be constructed in its half-neighborhood.

Let us consider the equation

$$
a(\lambda) x(t)=3 \int_{0}^{1} t s\left(x(s)+x^{2}(s)\right) d s
$$

1st case: $a\left(\lambda_{0}\right)=1, a^{\prime}\left(\lambda_{0}\right) \neq 0$.

Using theorem $2 \lambda_{0}$ is bifurcation point. Moreover, branching equation here is following

$$
-a^{\prime}\left(\lambda_{0}\right)\left(\lambda-\lambda_{0}\right) \xi+\frac{3}{5} \xi^{3}+\cdots=0
$$

and exists two small real solutions

$$
\xi_{ \pm}= \pm \sqrt{\frac{5}{3} a^{\prime}\left(\lambda_{0}\right)\left(\lambda-\lambda_{0}\right)}+o\left(\left(\lambda-\lambda_{0}\right)^{1 / 2}\right)
$$

as $a^{\prime}\left(\lambda_{0}\right)\left(\lambda-\lambda_{0}\right)>0$. Hence here $\lambda_{0}$ is strong bifurcation point. Moreover, integral equation in half-neighborhood of point $\lambda$ has two real solutions $x_{ \pm}(t) \sim \pm t \sqrt{\frac{5}{3} a^{\prime}\left(\lambda_{0}\right)\left(\lambda-\lambda_{0}\right)}$.

2nd case: $a\left(\lambda_{0}\right)=1, a^{\prime}\left(\lambda_{0}\right)=0, a^{\prime \prime}\left(\lambda_{0}\right) \neq 0$.

In this case conditions of Theorem 2 are not fulfilled. Moreover, if $a(\lambda) \neq 1$ as $\lambda \neq \lambda_{0}$ then integral equation apart from trivial solution has no other small real solutions. We demonstrated that trivial solution is isolated in this case.

Under conditions of Theorems 2, 3 equation $B x=R(x, \lambda)$ can have nontrivial solution depending on arbitrary small parameters only for $\lambda=\lambda^{*}$, where $\lambda^{*}$ is bifurcation point. 
Example 2. Let us consider the following integral equation

$$
(1+\lambda) x(t)=3 \int_{0}^{1} t s x(s) d s+a(t) \int_{0}^{1} x^{2}(s) d s
$$

Let $\int_{0}^{t} t a(t) d t=0$. Using Theorems 2 and 3 we can conclude that $\lambda=0$ is bifurcation point. All the solutions of this equation can be presented as follows

$$
x(t)=\frac{3 t}{1+\lambda} C_{1}+a(t) \frac{C_{2}}{1+\lambda_{1}}
$$

where $C_{1}=\frac{c_{1}}{1+\lambda}, C_{2}=\int_{0}^{1}\left(\frac{3 s}{1+\lambda} C_{1}+\frac{a(s)}{1+\lambda} C_{2}\right)^{2} d s$

Then there are two cases:

1st case. Let $\lambda \neq 0$. Then $c_{1}=0, c_{2}=\frac{1}{(1+\lambda)^{2}} \int_{0}^{1} a^{2}(s) d s c_{2}^{2}$. If one select $c_{2}=0$, then we get the trivial solution $x(t)=0$. If we assume $c_{2}=\frac{(1+\lambda)^{2}}{\int_{0}^{1} a^{2}(s) d s}$ then we get solutions of equations with no small as $\lambda \rightarrow 0$. Then $\lambda=0$ is unique bifurcation point.

2nd case. Let $\lambda=0$. Then $x(t)=3 t c_{1}+a(t) c_{2}$, and $c_{2}=3 c_{1}^{2}+c_{2}^{2} \int_{0}^{1} a^{2}(s) d s$. Hence in the second case equation has two c-parametric solutions

$$
x_{1,2}(t)=a(t) c \pm 3 t \sqrt{c-c^{2} \int_{0}^{1} a^{2}(s) d s}
$$

which are real for $0 \leq c \leq \frac{1}{\int_{0}^{1} a^{2}(s) d s}$. Obviusly $x_{1,2} \rightarrow 0$ as $c \rightarrow 0$

Let us consider one more model from mechanics.

Example 3. Let us consider the equation

$$
F(x, \lambda):=x(t)-2 \int_{0}^{\pi} \sum_{n=1}^{\infty} \frac{1+\lambda^{2 n}}{n\left(1+\lambda^{2 n}\right)} \cos n t \cos n s e^{x(s)} d s\left(\int_{0}^{\pi} e^{x(s)} d s\right)^{-1}=0
$$

Operator $F(x, \lambda)$ is differentiable wrt $x$ in sense of Fréchet and Theorems 2 and 3 can be applied. Here

$$
B(\lambda) x=x(t)-\frac{2}{\pi} \int_{0}^{\pi} \sum_{n=1}^{\infty} \frac{1-\lambda^{2 n}}{n\left(1+\lambda^{2 n}\right)} \cos n t \cos n s x(s) d s
$$

Operator $B(\lambda)$ for $\lambda \neq 0$ has inverse bounded. Then using Theorem 2 only point $\lambda=0$ is the only bifurcation point. Equation $B(0) x=0$ has nontrivial solution $\phi(t)=\cos t . B(\lambda)$ is self-adjoint operator. Branching equation corresponding bifurcation point $\lambda=0$ is following

$$
L(\xi, \lambda):=\xi \lambda^{2}(-2+r(\xi, \lambda))=0
$$

where $\xi(0,0)=0$. Then for $\lambda=0$ parameter $\xi$ remains arbitrary, and equation $F(x, 0)=0$ has nontrivial parametric solution

$$
x(t, \xi)=2 \sum_{n=1}^{\infty} \frac{\cos n t}{n}(\xi / 2)^{n}
$$

for $|\xi|<2$

For construction of parametric solutions in other simple cases it is usefull to use the following result. 
Lemma 3. Let $B \in \mathcal{L}(X \rightarrow Y)$ is Fredholm operator, $\left\{\phi_{i}\right\}_{1}^{n}$ is basis in $N(B),\left\{\psi_{i}\right\}_{1}^{n}$ is basis in $N\left(B^{*}\right), R(x)$ is nonlinear operator, $R(0)=0, R_{x}(0)=0$. Let $\left\langle R(x), \psi_{i}\right\rangle=0, i=1, \ldots, n$ for $\forall x$. Then equation $B x=R(x)$ has c-parametric small solution

$$
x=\sum_{i=1}^{n} c_{i} \phi_{i}+\Gamma u(c)
$$

for $c \rightarrow 0$. Here $\Gamma=\left(B+\sum_{1}^{n}\left\langle\cdot, \gamma_{i}\right\rangle z_{i}\right)^{-1}$ is bounded operator. Function $u(t)$ is constructed for small $|c|$ using method of successive approximations $u_{n}=R\left(\sum_{1}^{n} c_{i} \phi_{i}+\Gamma u_{n-1}\right), u(0)=0, n=1,2, \ldots$

Proof. First of all let us notice that $B \Gamma u=u$ if $\left\langle u, \phi_{i}\right\rangle=0, i=1, \ldots, n$. Then, taking into account conditions of the Lemma, we get the following equation to find $u$

$$
u=R\left(\sum_{i=1}^{n} c_{i} \phi_{i}+\Gamma u\right)
$$

The latter equation for sufficiently small $\left|c_{i}\right|, i=1, \ldots, n$ using the implicit operator theorem will enjoy unique continuous solution $u(c) \rightarrow 0$ and this solution can be found using successive approximations $u_{n}=R\left(\sum_{i=1}^{n} c_{i} \phi+\Gamma u_{n-1}\right), u_{0}=0$.

Example 4. Let us consider the equation

$$
x(t)-\int_{a}^{b} \sum_{i=1}^{n} a_{i}(t) a_{i}(s) x(s) d s=\int_{a}^{b} K(t, s) x^{2}(s) d s,
$$

where all the function are continuous,

$$
\int_{a}^{b} a_{i}(t) a_{j}(t) d t=\left\{\begin{array}{ll}
1, & i=j \\
0, & i \neq j
\end{array} .\right.
$$

Let $\int_{a}^{b} a_{i}(t) K(t, s) d t \equiv 0, i=1, \ldots, n$. Then conditions of Lemma 3 are fulfilled for $\phi_{i}=\psi_{i}=a_{i}(t)$. Moreover, operator $\Gamma$ appears to be an identity operator. Then the desired solution can be constructed as following sum $x=\sum_{i=1}^{n} c_{i} a_{i}(t)+u\left(t, c_{1}, \ldots, c_{n}\right)$, where function $u$ defined from eqaution

$$
u(t, c)=\int_{a}^{b} K(t, s)\left(\sum_{i=1}^{n} c_{i} a_{i}(s)+u(s, c)\right)^{2} d s
$$

using successive approximations for arbitrary $c_{1}, \ldots, c_{n}$ from some neighborhood of origin. In order to estimate this neighborhood of covergence, let us employ the method of convex majorants of L. V. Kantorovich [? ]. Let $\max _{a \leq t \leq b} \int_{a}^{b}|K(t, s)| d s=m$. We define the majorant system

$$
\left\{\begin{array}{l}
r=m(\rho+r)^{2} \\
1=2 m(\rho+r)
\end{array}\right.
$$

Then $r=\rho=1 / 4 m$.

Let vector $c=\left(c_{1}, \ldots, c_{n}\right)^{T}$ sutisfies the estimate

$$
\max _{a \leq t \leq b} \sum_{i=1}^{n}\left|c_{i}\right|\left|a_{i}(t)\right|<\frac{1}{4 m}
$$


Then sequence

$$
u_{m}(t, c)=\int_{a}^{b} K(t, s)\left(\sum_{i=1}^{n} c_{i} a_{i}(s)+u_{m-1}(s, c)\right)^{2} d s, u_{0}=0
$$

will converge. The desired $c$-parametric solution $x(t, c)$ satisfies the estimate $|x(t, c)|<\frac{1}{2 m}$.

\section{Solutions Parametrization and Iterations in Branch Points Neighborhood}

The objective of this section is to describe the iteration scheme with uniformization parameter selection and initial approximations of branches of solution of eq. (1). It is to be noted that in sec. 3 condition $R(0, \lambda)=0$ can be unsatisfied.

An important role of power geometry [38] and Newton diagram is well known in asymptotic analysis of finite-dimentional systems when implicite theorem's conditions are not fulfilled. Solution of operator equation (1) reduces to solution of such type finite-dimentional LS system.

The main stages of this approach we describe below. Similar with sec. 1 let us consider the equation

$$
B x=R(x, \lambda) \text {. }
$$

But now $\Lambda=\mathbb{R}^{1}$, operator $R(x, \lambda)=\sum_{i+k \geq 2} R_{i k}(x) \lambda^{k}+R_{01} \lambda$ is analytic in the neighborhood of origin, $R_{i k}(t x)=t^{i} R_{i k}(x), t \in \mathbb{R}^{1}, B$ is Fredholm operator. We have to construct the solution $x \rightarrow 0$ as $\lambda \rightarrow 0$. For iteration scheme construction one needs coefficients of branching LS system. Let us use the change

$$
x=\sum_{j=0}^{n} \xi_{s} \phi_{s}+\Gamma y(\xi, \lambda)
$$

where

$$
\left\langle y, \psi_{i}\right\rangle=0, i=1, \ldots, n
$$

and equation (13) will be converted to

$$
y=R(\xi \phi+\Gamma y, \lambda)
$$

Using the Implicit Operator Theorem for small ||$\xi||$ and $|\lambda|$ we have unique small solution

$$
y=\sum_{m \geq 2} y_{m 0}(\xi \phi)+\sum_{m \geq 0} \sum_{v \geq 1} y_{m v}(\xi \phi) \lambda^{v}
$$

Since $\xi \phi=\sum_{i=1}^{n} \xi_{i} \phi$ and $y_{m v}(\xi \phi)$ are $m$-homogenius in $\xi$, then for coefficient calculation we constuct reccurent formulae

$$
\begin{gathered}
y_{20}(\xi \phi)=R_{20}(\xi \phi), \\
y_{m 0}=\frac{1}{m !} \frac{d^{m}}{d \mu^{m}} \sum_{i \geq 2}^{m} R_{i 0}\left(\Gamma \sum_{i=1}^{m-1} y_{i 0} \mu^{i}\right)_{\mu=0}, m=3,4, \ldots, \\
\Gamma y_{10}:=\xi \phi, \\
y_{10}:=\sum_{i=1}^{n} \xi_{i} z_{i}, y_{01}=R_{01}, \\
y_{0 n}=\left.\frac{1}{n !} \frac{d^{n}}{d \lambda^{n}} \sum_{i+k \geq 2}^{n} R_{i k}\left(\Gamma \sum_{s=1}^{n-1} y_{0 s} \lambda^{s}\right) \lambda^{k}\right|_{\lambda=0}, n=2,3, \ldots, \\
y_{n-j, j}=\left.\frac{\partial^{n}}{(n-j) ! j !} \frac{1}{\partial \lambda j \partial \mu^{n-j}} \sum_{i+k \geq 2}^{n} \lambda^{k} R_{i k}\left(\Gamma \sum_{i+k \geq 1}^{k-1} y_{i k} \mu^{i} \lambda^{k}+\sum_{s=0}^{j-1} \Gamma y_{n-s, s} \mu^{n-s} \lambda^{s}\right)\right|_{\lambda=\mu=0}
\end{gathered}
$$


$n=2,3, \ldots, j=1, \ldots, n-1$. The sequence $\left\{y_{n}\right\}, y_{0}=0$ converges to solution (17), where

$$
y_{n}=R\left(\xi \phi+\Gamma y_{n-1}, \lambda\right), n=1,2, \ldots
$$

Substitution of solution (17) into (15) gives the following branching LS system

$$
L^{j}(\xi, \lambda):=\sum_{m \geq 2} L_{m 0}^{j}(\xi)+\sum_{m \geq 0} \sum_{v \geq 1} L_{m v}^{j}(\xi) \lambda^{v}=0, j=1, \ldots, n,
$$

where

$$
L_{m v}^{j}=\left\langle y_{m v}(\xi \phi), \psi_{j}\right\rangle=\sum_{m_{1}+\cdots+m_{n}=m} L_{m_{1}, \ldots, m_{n}, v}^{j} \xi_{1}^{m_{1}}, \ldots, \xi_{n}^{m_{n}}
$$

For symmetry let us put $\lambda=\xi_{n+1}$ in (18). Let $L\left(\xi_{1}, \ldots, \xi_{n+1}\right)$ be one of the left hand sides of system (18). Eliminate on the corresponding power $\xi_{i}$ for sake of clarity we assume

$$
L\left(\xi_{1}, \ldots, o_{i}, \ldots, \xi_{n+1}\right) \neq 0
$$

for $i=1, \ldots, n+1$. Let $\operatorname{supp} L=\left\{i \mid i \in \mathbb{N}_{+}^{n+1}, L_{i} \neq 0\right\}, \mathbb{N}_{+}$is set of positive integer numbers.

Let us introduce

Condition 1. We fix positive $\alpha_{1}, \ldots, \alpha_{n+1}, \theta_{1}, \ldots, \theta_{n}$ such as for $\xi_{i}=\varepsilon^{\alpha_{i}} v_{i}, i=1, \ldots, n+1$ and $\varepsilon \rightarrow 0$

$$
L^{j}=\varepsilon^{\theta_{j}}\left(l_{j}\left(v_{1}, \ldots, v_{n+1}\right)+r_{j}(\nu, \varepsilon)\right),
$$

where $l_{j}=\sum_{(i, \alpha)=\theta_{j}} L_{i}^{j} v^{i}, i=\left(i_{1}, \ldots, i_{n+1}\right), \alpha=\left(\alpha_{1}, \ldots, \alpha_{n+1}\right), v^{i}=v_{1}^{i_{1}}, \ldots, v_{n+1}^{i_{n+1}}, r_{i}(v, 0)=0$.

Let us now introduce the following

Definition 4. Hyperplane

$$
l:\left\{\xi \in \mathbb{R}_{+}^{n+1} \mid(\xi, \alpha)=\theta\right\}
$$

we call support plane for supp $L$, if

1. $(\xi, \alpha) \geq \theta$ for $\xi \in \operatorname{supp} L$,

2. $l \cap \operatorname{supp} L \neq 0$.

Then algebraic Condition 1 from geometrical point of view means that hyperplanes $l_{j}=\{\xi \in$ $\left.\mathbb{R}_{+}^{n+1} \mid(\xi, \alpha)=\theta_{j}\right\}, j=1, \ldots, n$ are correspondingly support hyperplanes for supp $L^{j}, j=1, \ldots, m$. In case of symmetry when $\alpha_{1}=\cdots=\alpha_{m}, \alpha_{m+1}=\cdots=\alpha_{n+1}$, the hyperplane $l_{j}$ is symmetric wrt axis $\xi_{1}, \ldots, \xi_{m}$ and axis $\xi_{m+1, \ldots, \xi_{n+1}}$. In such case for verification of Condition 1 the Newton digramm can be employed. The method of numbers $\alpha_{i}, \theta_{i}$ selection proposed by Bruno gaurantees the satisfaction of Condition 1 in the general case.

Condition 2. Let an algebraic system

$$
l_{j}\left(v_{1}, \ldots, v_{n+1}\right)=0, j=1, \ldots, n
$$

has solution $v^{0}=\left(v_{1}^{0}, \ldots, v_{n+1}^{0}\right)$, and for $v=v^{0}$

$$
\operatorname{det}\left[\frac{\partial l_{j}}{\partial v_{i}}\right]_{j=1, \ldots, n, i=(1, \ldots, n+1) \backslash *} \neq 0,
$$

where $(1, \ldots, n+1) \backslash *:=\left(1, \ldots, *-1, *+1, \ldots, n^{*}\right)$.

Solution $v^{0} \neq 0$ we call as full rank solution for system (20). Here index $*$ fixes rank minor of the matrix $\left[\frac{\partial l_{j}}{\partial v_{i}}\right]$ for $v=v^{0}$. 
Lemma 4. Let conditions 1 and 2 are fulfilled. Then branching system (18) has small solutions as $\varepsilon \rightarrow 0$

$$
\begin{gathered}
\xi_{i}=\varepsilon^{\alpha_{i}}\left(v_{i}^{0}+o(1)\right), i=(1, \ldots, n+1) \backslash *, \\
\xi_{*}=\varepsilon^{\alpha^{*}} v_{*}^{0},
\end{gathered}
$$

where $\xi_{n+1}:=\lambda(\varepsilon)$.

Proof follows from Implicit Function Theorem due to conditions 1 and 2. Using substitution of determined $\xi_{i}(\varepsilon), i=1, \ldots, n$ and $\lambda(\varepsilon)$ into (17) and taking into account (2) we get the desired pair $x(\varepsilon), \lambda(\varepsilon)$ satisfies equation (13). Then the following Theorem takes place

Theorem 4. Let conditions 1 and 2 be fulfilled. Then eq. (13) enjoys small solution $x=x(\varepsilon) \rightarrow 0 \lambda=$ $\lambda(\varepsilon) \rightarrow 0$ as $\varepsilon \rightarrow 0$.

System (20) can contain several solutions and choice of vectors $\alpha, \theta$ is not unique in general case, the eq. (13) can contain several solutions.

Let us consider the basic case on analytical calculation of the solution for eq. (13) using the method of undetermined coefficients. Let nonlinear system (20) is as follows

$$
l_{j}\left(v_{1}, \ldots, v_{n+1}\right): b_{j}\left(v_{1}, \ldots, v_{n+1}\right) \sum_{k=1}^{n+1} a_{j k} v_{k}=0, j=1, \ldots, n
$$

Here $\operatorname{rank}\left[a_{j k}\right]_{k=1, \ldots, n+1, j=1, \ldots, n}=n$. Then branching equation (18) call quasilinear. In this case it is easy to constract the asymptotic of solution for eq. (13). Indeed, fixing rank minor $\left[a_{j k}\right]_{k=(1, \ldots, n+1), j=1, \ldots, n}$ we can construct nontrivial solution $v^{0}=\left(v_{1}^{0}, \ldots, v_{n+1}^{0}\right)$ of the following system of linear algebraic equations

$$
\sum_{k=1}^{n+1} a_{j k} v_{k}=0, j=1, \ldots, n
$$

for selected $v_{*}^{0}$. Vector $v^{0}$ obviously satisfies system (20). Let us assume $b_{j}\left(v_{1}^{0}, \ldots, v_{n+1}^{0}\right) \neq 0, j=$ $1, \ldots, n$. Then, using Implicit Function Theorem, branching system (18) has small solution with asymptotics $v_{i} \sim \varepsilon^{\alpha_{i}} v_{i}^{0}, i=1, \ldots, n+1$. Using formulae (17), (2), we can conclude that asymptotics of the corresponding small solution of eq. (13) satisfies the following estimates

$$
\begin{gathered}
\lambda(\varepsilon) \sim \varepsilon^{\alpha_{n+1}} v_{n+1}^{0}, \\
P x(\varepsilon) \sim \sum_{i=1}^{n} \varepsilon^{\alpha_{i}} v_{i}^{0} \phi_{i}
\end{gathered}
$$

where $P:=\sum_{i=1}^{n}\left\langle\cdot, \gamma_{i}\right\rangle \phi_{i}$ is the projector on subspace $N(B)$. Under the certain conditions, analytical solution of eq. (13) can be effectively constructed using the method of undetermined coefficients as series $x(\lambda)=\sum_{i=1}^{\infty} x_{i} \lambda^{i}$

Indeed, let in eq. (13)

$$
\begin{gathered}
R(x, \lambda)=\sum_{k=1}^{\infty} R_{1 k} \lambda^{k} x+\sum_{i+k=3}^{\infty} R_{i k}(x) \lambda^{k}+\sum_{k=1}^{\infty} R_{0 k} \lambda^{k}, \\
\operatorname{det}\left[\left\langle R_{11} \phi_{k}, \psi_{i}\right\rangle\right]_{k=1, \ldots, n} \neq 0, \\
\left\langle R_{01}, \psi_{i}\right\rangle=0, i=1, \ldots, n .
\end{gathered}
$$


Then branching equation will be quasilinear for $\alpha_{1}=\cdots=\alpha_{n+1}=1, p=2$. Solution of eq. (13) we construct as series $x=\sum_{i=1}^{\infty} x_{i} \lambda^{i}$. For calculation of the coefficients $x_{i}$ using method of undetermined coefficients we obtain the following recurrent sequence of linear equations

$$
\begin{gathered}
B x_{1}=R_{01} \\
B x_{2}=R_{11} x_{1}+R_{02} \\
B x_{m}=R_{11} x_{m-1}+f_{m}\left(x_{1}, \ldots, x_{m-2}\right), m=3,4, \ldots
\end{gathered}
$$

Hence, $x_{1}=\sum_{i=1}^{n} c_{1 i} \phi_{i}+\Gamma R_{01}$, where $\Gamma=\left(B+\sum_{i=1}^{n}\left\langle\cdot, \gamma_{i}\right\rangle z_{i}\right)^{-1}$. Vector $c_{1}=\left(c_{11}, \ldots, c_{1 n}\right)^{\prime}$ can be uniquely defined from the following system of linear algebraic equations

$$
\sum_{k=1}^{n}\left\langle R_{11} \phi_{k}, \psi_{i}\right\rangle c_{1 k}+\left\langle R_{11} \Gamma R_{01}, \psi_{i}\right\rangle+\left\langle R_{02}, \psi_{i}\right\rangle=0, i=1, \ldots, n
$$

which corresponds to the resolving conditions of the 2nd equation of the sequence. Similarly, $x_{m}=$ $\sum_{i=1}^{n} c_{1 n} \phi_{i}+\hat{x}_{m}$, where vector $c_{m}=\left(c_{m+1, \ldots, c_{m+n}}\right)^{\prime}$ is defined from the system of linear algebraic equations, element $\hat{x}_{m}$ we uniquely construct in the subspace $X_{\infty-n}$ using operator $\Gamma$.

As result, the following statement can be formulated concerning the existence and construction of the analytical solution of eq. (13).

$$
\text { Let } R(x, \lambda)=\sum_{k=p}^{\infty} R_{1 k} \lambda^{k} x+\sum_{i+k=p+2} R_{i k}(x) \lambda^{k}+\sum_{k=1}^{\infty} R_{0 k} \lambda^{k}, \operatorname{det}\left[\left\langle R_{1 p} \phi_{i}, \psi_{k}\right\rangle\right]_{i, k=1, \ldots, n} \neq 0,
$$

$\left\langle R_{0 k}, \psi_{i}\right\rangle=0, k=1, \ldots, p, i=1, \ldots, n$. Then eq. (13) enjoys an analytical solution $x=\sum_{i=1}^{\infty} x_{i} \lambda^{i}$, where $x_{i}=\sum_{k=1}^{n} c_{i k} \phi_{k}+\hat{x}_{i}, i=1,2, \ldots$ Vectors $c_{i}=\left(c_{i 1}, \ldots, c_{i n}\right)^{\prime}$ can be uniquely calculated from the system of linear algebraic equations with matrix $\left[\left\langle R_{1 p} \phi_{i}, \psi_{k}\right\rangle\right]_{i, k=1, \ldots, n}$, elements $\hat{x}_{i}$ can be determined in the subspace $X_{\infty-n}$ uniquely.

\section{N-step iteration scheme for construction of the solution of equation (13)}

Let conditions 1 and 2 are fulfilled for branching system (18) and $\xi^{0}$ is solution of system (20) is full rank solution. Let $r=\min \left(\alpha_{1}, \ldots, \alpha_{n+1}\right)$. Solution $x(\varepsilon), \lambda(\varepsilon)$ of equation (13) we seek in the form

$$
x(\varepsilon)=\xi(\varepsilon) \phi+\Gamma \varepsilon^{r} y(\varepsilon), \lambda(\varepsilon)=\varepsilon^{\alpha_{n+1}} \xi_{n+1}(\varepsilon),
$$

where $\xi(\varepsilon) \phi:=\sum_{i=1}^{n} \varepsilon^{\alpha_{i}} \xi_{i}(\varepsilon), \xi_{i}(0)=v_{i}^{0}, i=1, \ldots, n+1$.

$$
y(0)= \begin{cases}0, & r<\alpha_{n+1}, \\ R_{01} v_{n+1}^{0}, & r=\alpha_{n+1} .\end{cases}
$$

Lets put in $(21) \xi_{*}=v_{*}^{0}$. The rest $\xi_{i}(\varepsilon), i \neq *$ and $y(\varepsilon)$ continuous in zero, we define from system

$$
\varepsilon^{r} y=R\left(\xi(\varepsilon) \phi+\Gamma \varepsilon^{r} y, \varepsilon^{\alpha_{n+1}} \xi_{n+1}(\varepsilon)\right):=\Phi\left(\varepsilon^{e} y, \xi, \varepsilon\right),
$$

with condition

$$
\left\langle y, \psi_{i}\right\rangle=0, i=1, \ldots, n .
$$

Let us outline that $\lim _{\varepsilon \rightarrow 0} \varepsilon^{-r} \Phi\left(\varepsilon^{r} y, \xi, \varepsilon\right)=y\left(0\right.$.) Because $\xi_{*}(\varepsilon) \equiv v_{*}^{0}$ then in system (22) - (23) functions $y(\varepsilon), \xi_{i}(\varepsilon)$ are unknown for $i \neq *$. System $(22)-(23)$ consists of $(n+1)$ equations with $(n+1)$ unknowns. 
Let us transfer system (22) - (23) in order to meet the conditions of Implicite Operator Theorem in the neighborhood of point $y(0), v_{I}^{0}, i \neq \star$. Then by introduction of iterative process in system (22) (23), we get the following system

$$
\begin{gathered}
y=\varepsilon^{-r} \underbrace{\Phi(\Phi \ldots(\Phi \ldots))}_{N}:=\varepsilon^{-r} \Phi^{N}\left(\varepsilon^{r} \Gamma y, \xi(\varepsilon)\right), \\
\varepsilon^{\theta_{j}}\left\langle R\left(\xi(\varepsilon) \phi+\Gamma \Phi^{N}\left(\varepsilon^{r} \Gamma y, v, \varepsilon\right), \varepsilon^{\alpha_{n+1}} v_{n+1}\right), \psi_{j}\right\rangle=0, j=1, \ldots, n .
\end{gathered}
$$

Here $v_{*}(\varepsilon)=v_{*}^{0}$.

$$
\Phi^{N}:=R\left(\xi(\varepsilon) \phi+\Gamma R\left(\xi \phi+\cdots+\Gamma R\left(\xi \phi+\Gamma \varepsilon^{r} y, \xi_{n+1}\right), \ldots, \xi_{n+1}\right)\right.
$$

Any solution $y, v_{i, i \neq *}$ satisfying system (24) - (25) satisfies also (22) - (23) . System (24) - (25) we conider as single operator equation

$$
K(u, \varepsilon)=0,
$$

where $K: E \times \mathbb{R}^{1} \rightarrow E, E=Y \times \mathbb{R}^{n},\|u\|:=|| y \|+|\xi|, u=$ $\left(y(\varepsilon), \xi_{1}(\varepsilon), \ldots, \xi_{*-1}(\varepsilon), \xi_{n+1}(\varepsilon), \ldots \xi_{n+1}(\varepsilon)\right)^{T}, \xi_{*}(\varepsilon) \equiv v_{*}^{0}$. Using conditions 1 and 2 for $N>\max _{1 \leq j \leq n}\left(\theta_{j}-r\right)$ operator $K$ will be continuous in the neighborhood of the point $u_{0}=\left(y(0), \xi^{0}\right)$, $\varepsilon=0, K\left(u_{0}, 0\right)=0$ and exists Fréchet derivative $K_{u}(u, \varepsilon)$ continuous in point $\left(u_{0}, 0\right)$,

$$
K_{u}\left(u_{0}, 0\right)=\left(\begin{array}{cc}
I & 0 \\
0 & D
\end{array}\right)
$$

Here matrix $D=\left[\frac{\partial l_{j}\left(\xi^{0}\right)}{\partial \xi_{i}}\right]$ is rank minor of matrix $\left[\frac{\partial l_{j}}{\partial v_{i}}\right]$ on solution $v^{o}$ of algebraic system (20).

Due to Condition 2 det $D \neq 0$ and there exists bounded inverse operator

$$
K_{u}^{-1}\left(u_{0}, 0\right)=\left(\begin{array}{cc}
I & 0 \\
0 & D^{-1}
\end{array}\right)
$$

Then equation (26) satisfies all the conditions of Implicite Operator Theorem, equation (26) enjoys unique continuous solution $u(\varepsilon)$ such as

$$
u_{m}=u_{m-1}-K^{-1}\left(u_{0}, 0\right) K\left(u_{m-1}, \varepsilon\right)
$$

converges to this solution in the neighborhood of the point $\varepsilon=0$. Therefore, if conditions 1 and 2 are fulfilled, then equation (26) enjoys small solution $x=x(\varepsilon) \rightarrow 0, \lambda=\lambda(\varepsilon) \rightarrow 0$ as $\varepsilon \rightarrow 0$, where

$$
\operatorname{Px}(\varepsilon)=\sum_{i=1}^{n} \varepsilon^{\alpha_{i}}\left(v_{i}^{0}+o(1)\right) \phi_{i}
$$

$\lambda(\varepsilon) \sim \varepsilon^{\alpha_{n+1}} v_{n+1}^{0}$. Moreover, sequences

$$
\begin{gathered}
x_{m}=\sum_{i=1}^{n} \varepsilon^{\alpha_{i}} \xi_{i}^{m}(\varepsilon)+\Gamma \varepsilon^{r} y_{m}, \\
\lambda_{m}=\varepsilon^{\alpha_{n+1}} \xi_{n+1}^{m}(\varepsilon), m=1,2, \ldots,
\end{gathered}
$$

where $y_{m}, \xi_{i}^{m}(\varepsilon), i=1, \ldots, n+1$ are defined iterations (27) converges as $m \rightarrow \infty$ to desired small solution $x(\varepsilon) \rightarrow 0, \lambda(\varepsilon) \rightarrow 0$ as $\varepsilon \rightarrow 0$. Right hand sides of formulae (21), (22) used in iterations (27) 
we build an inverse operator $\Gamma$. Therefore for element $x_{m}$ calculation it is necessary to solve $N$-linear equations

$$
\left(B+\sum_{i=1}^{n}\left\langle\cdot, \gamma_{i}\right\rangle z_{i}\right) x=f
$$

with continuosly invertiable operator. Therefore, proposed iteration scheme is $N$-step on each step.

\section{Remarks, regularization and generalizations}

The right hand side of the iteration scheme (27) contains operator $\Gamma$ introduced by V.A. Trenogin [39] and negative powers of the small parameter $\varepsilon$. But this singularity is resolvable. Indeed, in case of polynomial nonlinearity wrt negative powers of $\varepsilon$ one can eliminate the corresponding powers of parameter $\varepsilon$. For more details readers may refer to [9,30,31]. Then, taking into account boundness of operator $\Gamma$ and its regularizing properties $[23,39]$ convergence of proposed $N$-steps method of successive approximations will be uniform in the branch point's neighborhood. If it is not possible to perform explicite eliminations, then for sake of stable computations in case of negative powers of $\varepsilon$ one can employ the change of $\varepsilon$ onto $\varepsilon+\operatorname{sign} \varepsilon \delta^{v}$, where $0<v<1 / 2 p, p=\max _{1 \leq j \leq n}\left(\theta_{j}-r\right)$, where $\delta$ is maximal absolute error of computations. Then proposed iteration scheme can be classified as Tikhonov-Lavrentiev regularisation algorithm.

Finally, let us outline that in number of applications Condition 2 for branching system is not satisfied. Analysis of corresponding branching solutions depending on free parameters linked with model's symmetry requires methods from [23,25,26,31]. Usually, in such cases it is assumed the existence of linear bounded operators $S \in \mathcal{L}(X \rightarrow X)$ and $K \in \mathcal{L}(Y \rightarrow Y)$ such as $B S=K B$, $R(S X, \lambda)=K R(x, \lambda)$ for $\forall x, \lambda \in \Omega$.

Operators $S, K$ can be projectors. If problem $G$-invariant then $S, K$ can be parametric representations of $G$-group. In that case we say that eq. $(13)$ is $(S, K)$-interwined. In papers $[24,26]$ the iterative approach is implemented and developed using ideas of analytical method of Lyapunov-Schmidt in case of $(S, K)$-interwined equations. In this case it is allowed to change the parameter of uniformization of solutions branches.

Because of symmetry (13) with respect to main representatives of rotation group can be employed to transfer to the spheric coordinates and construct the solution depending on free parameters.

Example 5. Let us consider the equation

$$
x=\frac{1}{\pi} \int_{0}^{2 \pi} \cos (t-s) x(s) d s+\lambda x(t)+x^{2} .
$$

Here $\phi_{1}=\frac{\cos t}{\sqrt{\pi}}, \phi_{2}=\frac{\sin t}{\sqrt{\pi}}$ and $\Gamma$ is identity operator. Let us seek the small solution as $\lambda \rightarrow 0$ in form $x=\xi_{1} \phi_{1}+\xi_{2} \phi_{2}+y$, where $\int_{0}^{2 \pi} y(t) \phi_{i}(t) d t=0, i=1,2$. In the polar coordinates $\xi_{1}=\rho \cos \alpha, \xi_{2}=\rho \sin \alpha$, Branching LS system can be presented as follows

$$
\left(\rho \frac{\lambda}{1-\lambda}+\frac{3}{2 \pi} \frac{\rho^{3}}{(1-\lambda)^{5}}+r(\rho, \lambda)\right)\left(\begin{array}{c}
\cos \alpha \\
\sin \alpha
\end{array}\right)=0 .
$$

Here $r(\rho, \lambda)$ is an analytical function in the neighborhood of origin, and $r(\rho, \lambda)=o\left(\rho^{5}\right)$. Then the desired implicit parametrization of small solution is following

$$
\begin{gathered}
x=\rho \frac{\cos (t-\alpha)}{\sqrt{(} \pi)}+\mathcal{O}\left(\rho^{3}\right), \\
\lambda=-\frac{3}{2 \pi} \rho^{3}+\mathcal{O}\left(\rho^{4}\right),
\end{gathered}
$$


where $\alpha \in(-\infty, \infty)$ as $\rho \rightarrow 0$. After transition to explicit parametrization we can get two $\alpha$-parametric small solutions $x_{ \pm}= \pm \sqrt{-\frac{2 \lambda}{3}} \cos (t-\alpha)+o\left(|\lambda|^{1 / 2}\right)$ which are real valued as $\lambda \rightarrow-0$.

\section{Conclusion}

Results of this paper enable applications of the existence theorems for bifurcation points of nonlinear BVP problems and make it possible to construct an appropriate solutions. Our method has been also applied for solution of degenerate operator-differential and integral equations [17,18,21,23, 32], [37].

Problem of optimal uniformization parameters selection needs to take into account an insight of the problems and it is not yet solved in algebraic form. As footnote, let us outline the formulation and proof of the nonlocal theorems of existence of branching solutions in nonstandart models remains an important problem. For solution of these problems the Trenogin's nonlocal theorems from [6,7] can be employed.

Funding: This work is supported by RSF.

Conflicts of Interest: The authors declare no conflict of interest.

\section{References}

1. Lyusternik, L. A. Some questions of non-linear functional analysis (Russian). Usp. Mat. Nauk. 1954, 11, No. 6 (72), 145-168.

2. Krasnosel'skii, M.A. Some problems of nonlinear analysis, Uspekhi Mat. Nauk (Russian), 1954, 9:3 (61), 57-114.

3. Vainberg, M.M., Trenogin, V.A. The methods of Lyapunov and Schmidt in the theory of non-linear equations and their further development, Russian Math. Surveys, 1962, 17:2, 1-60. DOI: 10.1070/RM1962v017n02ABEH001127

4. Vainberg, M.M.; Trenogin, V.A. Theory of Branching of Solutions of Non-linear Equations, Noordhoff, 1974.

5. Sidorov, N.A. The optimal choice of initial approximations to solutions of regularized equations in the theory of branching, Math. Notes, 1976, 20:2, 710-713. DOI: 10.1007/BF01155880.

6. Trenogin, V.A. Lyusternik's theorem and the best parametrization of solutions to nonlinear equations, Funct. Anal. Appl., 1998, 32:1, 68-71. DOI: 10.1007/BF02465765

7. Trenogin, V.A. Locally Invertible operators and the method of continuation with respect to parameter, Funct. Anal. Appl., 1996, 30:2, 147-148. DOI: 10.1007/BF02509460

8. Loginov, B.V, Sidorov, N.A., Trenogin, V.A. Bifurcation, potentiality, group-theoretic and iterative methods. ZAMM 1996, 76(2), 245-248

9. Sidorov, N.A.; Loginov, B.V.; Sinitsyn, A.V.; Falaleev, M.V. Lyapunov-Schmidt methods in nonlinear analysis and applications, Kluwer Academic Publisher, 2002.

10. Sidorov, N.A., Trenogin, V.A. Bifurcation points of solutions of nonlinear equations, Nonlinear analysis and nonlinear differential equations (Russian), FizMatLit, Moscow, 2003, 5-49.

11. Markov, Yu., Rudykh, G., Sidorov, N., Sinitsyn, A., Tolstonogov, D. Steady-state solutions of the Vlasov-Maxwell system and their stability, Acta Appl. Math., 1992, 28:3, 253-293. DOI: 10.1007/BF00047089

12. Sidorov, D., Sidorov, N. Convex majornants method in the theory of nonlinear Volterra equations, Banach J. Math. Anal. 2012, 6(1), 1-10. DOI: 10.15352/bjma/1337014661

13. Loginov B.V., Konopleva, I.V., Rusak, Yu. B. Symmetry and potentiality in a general problem in bifurcation theory, Russian Math. (Iz. VUZ), 2006, 50:4, 28-38.

14. Karasozen, B., Loginov, B.V. Invariant reduction of partially potential branching equations and iterative methods in the problem on a bifurcation point with a symmetry, Differ. Equ., 2004, 40:3, 410-419. DOI: 10.1023/B:DIEQ.0000035778.88884.c7

15. Keller, H.B. Interaction, perturbation and muliplication in nonlinear bifurcation problems. Accad. Rad. Mech. 1972, 48, 83-108. 
16. Loginov, B.V., Sidorov, N.A. Group symmetry of the Lyapunov-Schmidt branching equation and iterative methods in the problem of a bifurcation point, Math. USSR-Sb., 1992, 73:1, 67-77. DOI: $10.1070 \% 2 F S M 1992 v 073$ n01ABEH002535

17. Sidorov, D. Integral dynamical models: singularities, signals \& control, vol. 87, World Scientific Series on Nonlinear Science Series A, edt. Leon O. Chua, World Scientific Publ. Pte Ltd, Singapore, 2015. DOI: $10.1142 / 9278$

18. Sidorov, D.N., N. A. Sidorov, N.A. Solution of irregular systems of partial differential equations using skeleton decomposition of linear operators, Vestnik YuUrGU. Ser. Mat. Model. Progr., 2017, 10:2, 63-73. DOI: $10.14529 / \mathrm{mmp} 170205$

19. Sidorov, N.A., Trufanov, A.V. Nonlinear operator equations with a functional perturbation of the argument of neutral type Differential Equations, 2010. 45, 1840-1844. DOI: 10.1134/S0012266109120155

20. Sidorov, N. A., Sidorov, D. N., Krasnik, A. V. Solution of Volterra Operator-integral equations in the nonregular case by the successive approximation method, Differential Equations, 2010, 46, 882-891. DOI: 10.1134/S001226611006011X

21. Sidorov, N.A. , Sidorov, D.N. On the solvability of a class of Volterra operator equations of the first kind with piecewise continuous kernels. Math. Notes. 2014 96(5), 811--826. DOI: 10.1134/S0001434614110170

22. Sidorov, D.N. Existence and blow-up of Kantorovich principal continuous solutions of nonlinear integral equations, Differential Equations. 2014, 50:9, 1217-1224. DOI: 10.1134/S0012266114090080

23. Sidorov, N., Sidorov, D., Sinitsyn, A. Toward general theory of differential-operator and kinetic models, vol. 97, World Scientific Series on Nonlinear Science Series A, edt. Leon O. Chua, World Scientific Publ. Pte Ltd, Singapore, 2020. DOI: 10.1142/11651.

24. Sidorov, N.A. Parametrization of simple branching solutions of full rank and iterations in nonlinear analysis. Russian Math. (Iz. VUZ). 2001. 45(9), 55-61. Zbl 1002.47042

25. Sidorov, N.A., Abdullin, V.R. Interlaced branching equations in the theory of non-linear equations. Sb. Math. 2001. 192(7), 1035-1052. DOI: 10.1070\%2FSM2001v192n07ABEH000582

26. Abdullin, V.R., Sidorov, N.A. Interlaced equations in branching theory. Dokl. Akad. Nauk. 2001, 377(3), 295-297. DOI: https:/ / doi.org/10.1070\%2FSM2001v192n07ABEH000582

27. Rendon, L., Synitsyn, A.V., Sidorov, N.A. Bifurcation points of nonlinear operators: existence theorems, asymptotics and application to the Vlasov-Maxwell system. Rev. Colombiana Mat. 2016 50(1), 85-107. DOI: 10.15446/recolma.v50n1.62200

28. Sidorov, N.A., Leontiev, R. Yu., Dreglea, A.I. On small solutions of nonlinear equations with vector parameter in sectorial neighborhoods. Math. Notes 2012 91(1), 90-104. DOI: 10.1134/S0001434612010105

29. Sidorov, N.A., Sinitsyn, A.V. On bifurcation points of the stationary Vlasov-Maxwell system with bifurcation direction. Progress in Industrial Mathematics at ECMI 98 (Gothenburg), European Consort. Math. Indust., Teubner, Stuttgart 1999, 295-302.

30. Sidorov, N.A. An N-step iterative method in the theory of the branching of solutions of nonlinear equations. Siberian Math. J. 1998 38(2), 330-341. DOI: 10.1007/BF02674632

31. Sidorov, N. A. Explicit and implicit parametrizations in the construction of branching solutions by iterative methods, Sbornik: Mathematics. 1995 186:2, 297-310. DOI: 10.1070\%2FSM1995v186n02ABEH000017

32. Sidorov, N.A., Sidorov, D.N. Solving the Hammerstein integral equation in the irregular case by successive approximations, Siberian Math. J., 2010, 51:2, 325-329. DOI: 10.1007/s11202-010-0033-4

33. Sidorov, N. A., Sidorov, D. N. Existence and construction of generalized solutions of nonlinear Volterra integral equations of the first kind. Differential Equations, 2006, 42, 1312-1316. DOI: 10.1134/S0012266106090096

34. Sidorov, D. N. Generalized solution to the Volterra equations with piecewise continuous kernels,Bulletin of the Malaysian Mathematical Sciences Society, 2014, 37:3, 757-768.

35. Sidorov, D. N., Sidorov, N.A. Generalized solutions in the problem of dynamical systems modeling by Volterra polynomials, Autom. Remote Control, 2011, 72:6, 1258-1263. DOI: 10.1134/S0005117911060130

36. Ovsyannikov, L.V. Group analysis of differential eqations Nauka: Moscow, 1987 (in Russian). Trans.: Academic Press, New York, 1992.

37. Sviridyuk G. A., Fedorov V. E. Linear Sobolev type equations and degenerate semigroups of operators, VSP, Utrecht, 2003.

38. Bruno, A. D. Power Geometry in algebraic and differential equations. Elsevier. 2000. 
39. Trenogin, V.A. Analyse fonctionelle, 1985, Moscow, Mir Publ. 